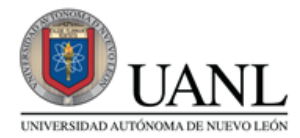

FACPYA
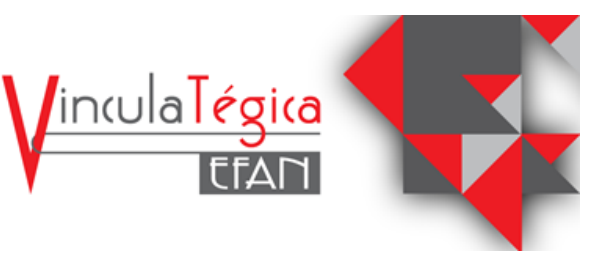

\title{
El impacto del home office y el balance de vida y trabajo en la retención de personal de la Generación Y durante el Covid-19
}

\author{
Ruth Isela Martínez Valdez ${ }^{1}$, Adriana Segovia Romo² y Katia Site Pérez Martínez ${ }^{3}$ \\ ${ }^{1}$ Universidad Autónoma de Nuevo León, ruth.martinezvld@uanl.edu.mx ,Pedro de Alba S/N Niños Héroes, \\ Ciudad Universitaria, 8112126749 \\ ${ }^{2}$ Universidad Autónoma de Nuevo León, adrianasegovia@hotmail.com, Pedro de Alba S/N Niños Héroes, \\ Ciudad Universitaria, 8114743621 \\ ${ }^{3}$ Universidad Autónoma de Nuevo León, katiasiteper@hotmail.com, Pedro de Alba S/N Niños Héroes, \\ Ciudad Universitaria, 8111815671
}

Información del artículo revisado por pares

Fecha de aceptación: junio-2021

Fecha de publicación en línea: diciembre-2021

DOI: https://doi.org/10.29105/vtga7.1-88

\section{Resumen}

Con la evolución de la tecnología y la declaración de la pandemia, el home office se ha convertido en una pieza clave en las organizaciones, aunado a ellos otro factor importante es el balance entre vida y trabajo, puesto que los roles laborales y personales pueden entrar en conflicto, además las organizaciones han dejado de lado programas para lograr un equilibrio entre la vida personal y laboral lo que impacta la retención laboral. La presente investigación pretende determinar el impacto del home office y el balance entre vida y trabajo en la retención del personal en hombres y mujeres de la generación "Y". El enfoque utilizado en esta investigación fue de corte cuantitativo con un diseño no experimental y transversal, de alcance descriptivo. La muestra fue de tipo no probabilístico incidental. Los participantes en este estudio fueron 106 jóvenes entre 24 y 28 años, de los cuales el 51\% fueron mujeres y el $49 \%$ fueron hombres pertenecientes a la Generación "Y", con trabajo actualmente. Se elaboró un instrumento tipo cuestionario cuyo nombre es "Factores de retención de personal" mismo que tiene 37 items con un $\alpha=0.958$, para este estudio se tomaron 3 variables dando un total de 19 ítems con un $\alpha=0.917$. Se realizó una prueba T-Student cuyos resultados fueron significativos para la variable home office con respecto a la retención de personal, caso contrario fue el resultado de balance entre vida y trabajo en donde no se encontró significancia con la variable de retención de personal a la retención del personal.

Palabras clave: Home Office, Balance Vida y Trabajo, Retención Laboral, Generación "Y", Género.

\footnotetext{
Abstract

With the evolution of technology and coupled with the declaration of the pandemic, the home office has become a key piece in organizations, coupled with them another important factor is the balance between life and work since work and personal roles can come into conflict and with the pandemic, the policies implemented for this purpose have stopped, thus affecting job retention. This research aims to determine the impact of the home office and the life balance on
} 
staff retention in men and women of the "Y" generation. The approach used in this research was quantitative with a non-experimental and cross-sectional design, descriptive in scope. The sample was of the incidental non-probabilistic type. The participants in this study were 106 young people between 24 and 28 years old, of which $51 \%$ were women and $49 \%$ were Men belonging to Generation "Y" with work. A questionnaire-type instrument was developed whose name is "personnel retention factors",

\section{Introducción}

Hoy en día el mundo está colapsado, se vive de una forma diferente a raíz de la aparición del COVID-19 y la declaración de la pandemia en el mundo laboral también hay cambios se abrió la posibilidad de nuevas formas de trabajar para poder seguir adelante, una de ellas es el home office, antes de la pandemia se hablaba de los beneficios que había para las empresas que lo practicaban, pero tras meses de estar trabajando desde casa (home office) surgen los efectos negativos como la falta del balance que existe entre la vida personal y la laboral (Martín y Reyes, 2020).

A causa del confinamiento en casa por la pandemia de COVID-19 las empresas buscan soluciones para salir adelante y el home office fue una de ellas, con esta modalidad se mantiene la distancia social y contener contagios, las personas se mantienen comunicados $y$ conectados, se le da continuidad a las operaciones y las personas se mantienen en sus puestos de trabajo, los empleados están dispuestos a sacrificar parte de su tiempo personal para conservar su trabajo y con ellos las organizaciones han logrado mantener un buen índice de retención del talento, especialmente en los jóvenes de la Generación Y que se caracteriza por buscar la flexibilidad en el trabajo para permanecer en él, para estos jóvenes lograr un balance entre su vida personal y laboral es significativo para hacer carrera en su organización actual, además que son nativos digitales y para ellos el home office es algo que les facilita manejar sus tiempos, algo que valoran. (Fócil, 2020; Martín y Reyes, 2020). which is made up of 37 items with $\alpha=0.958$. For this study, 3 variables were taken, giving a total of 19 items with $\alpha=0.917$. The findings found are quite interesting in which one stands out that there is a positive significance in the home office variable with respect to staff retention.

Keywords: Home Office, Work-Life Balance, Job Retention, Generation "Y", Gender.

Jel: M1, M12

Ahora bien, el uso de la tecnología ha permitido que las empresas logren ambientes más productivos y así sobrevivir a los tiempos de pandemia, sin embargo, esto obliga a las personas a estar confinadas en su hogar, en consecuencia, los trabajadores están perdiendo parte de su vida personal, ya que su vida laboral está ubicada en el mismo espacio, además, los trabajadores tienen problemas para organizar sus actividades y encontrar un equilibrio entre ambos mundos, debido a lo antes mencionado, es difícil mantener la energía para trabajar en un entorno donde hay situaciones externas que distraen su atención, además de lo cansado de estar muchas horas detrás de una pantalla (Álvarez, 2020).

De acuerdo con la Organización para la Cooperación y el Desarrollo Económico (OECD, 2020) en México existe el índice más bajo en cuestiones de Balance entre vida y Trabajo de 40 países analizados tiene una calificación de 1.1 de un rango de 0 a 10 obteniendo el lugar 39. Entre los criterios que se toman en cuenta está el de las horas laboradas, los mexicanos trabajan en promedio 2,148 horas al año es decir 41 horas a la semana con respecto a otros países que laboran 1,734 horas en promedio es decir 33 horas, el $28.7 \%$ de la población en México trabaja más de 50 horas cuatro veces más que el promedio. Demasiadas horas de trabajo es perjudicial para la salud, se pone en riesgo la seguridad y aumenta el estrés, esto es un desafío para buscar una mejor calidad de vida.

Ante la situación que se vive en nuestro país y debido al confinamiento provocado por la pandemia las empresas han tenido que suspender las prácticas establecidas con anterioridad que procuraban el balance entre vida y trabajo que, 
orillándolos a buscar soluciones para esta situación, y es que debido a las prácticas de home office tener un equilibrio entre la vida personal y el trabajo se ha convertido en el principal reto de los trabajadores en México y en el mundo, la carga de trabajo excesiva, la brecha de género que se presenta en las mujeres con las tareas del cuidado de la casa y de los hijos y la calidad del tiempo libre ha provocado un desequilibrio que afecta el desempeño de las personas, en México el 54\% de las mujeres que están haciendo home office manifiesta se encuentra en esta situación. (Tello y Vargas, 2020).

El objetivo general de esta publicación es determinar el impacto de las variables home office y balance entre vida y trabajo en la retención de personal entre hombres y mujeres de la generación Y durante el COVID-19

Las hipótesis de investigación son:

H1. El home office tiene una relación positiva con la retención del personal entre hombres y mujeres de la generación Y durante el Covid-19.

H2. El balance entre vida y trabajo tiene una relación positiva con la retención de personal entre hombres y mujeres de la generación $Y$ durante el COVID-19

\section{Marco Teórico}

\subsection{Home office}

Como consecuencia de la declaración de pandemia por COVID-19 en México y el resto del mundo, cambió la forma tradicional de trabajar, ante la emergencia sanitaria las organizaciones están en la búsqueda de soluciones a los problemas que se les presentan, se implementaron prácticas de home office, lo cual se ha convertido en una tendencia de la gestión del personal esto trajo beneficios, pero también desventajas y limitaciones, las organizaciones enfrentan retos, tales como: dotar del equipo y la tecnología a los trabajadores, definir horarios de trabajo y ofrecer capacitación a quien la necesite (OECD, 2020). Para Fócil (2020) el home office en una forma de trabajo a distancia que permite generar un balance entre la vida laboral y personal de los empleados de una empresa, que mejora la calidad de vida e impacta positivamente en la eficiencia.
Por otro lado, cabe mencionar que existen diversas teorías asociadas al home office, entre ellas se encuentra la teoría de empresa flexible que plantea que las organizaciones están planeando el empleo del futuro, centrados en el empleado y su conocimiento, capacidad y habilidad en el manejo de la tecnología para poder realizar sus actividades laborales desde un lugar diferente a las instalaciones de las organizaciones, esto con el apoyo de la empresa ofreciendo a su gente realizar sus actividades en la distancia salvaguardando su seguridad (Atkinson, 1987).

Con respecto a lo anterior, cabe señalar que en México se aprobaron reformas en el 2019 a la Ley Federal del Trabajo con respecto al home office, en el cual se busca garantizar un trabajo decente e igual entre remuneración, capacitación y seguridad social, en esta reforma se adicionó un segundo párrafo al artículo 311 de la LFT (2019) en el cual se define el teletrabajo como el trabajo que se realiza en el domicilio del trabajador o en algún lugar elegido por él, sin vigilancia del patrón, este trabajo se realiza a distancia usando las tecnologías de la información y comunicación. Según el Statista (2020) durante los meses de julio-agosto 2020 el $72 \%$ de los mexicanos estuvieron trabajando en casa durante el confinamiento, sin embargo y ante la nueva reforma solo 2 de cada 10 empresas están preparadas para poder seguir ofreciendo esta práctica cumpliendo con lo que exige la reforma.

\subsection{Balance entre vida y trabajo}

Aunque el entorno laboral sufrió cambios significativos con la declaración de la pandemia por COVID-19, los cuales ya venían presentándose de tiempo atrás debido al estrés del home office y al conflicto que se genera entre la vida laboral y la personal (Candela y Dabos, 2017). El tema del balance entre vida y trabajo ha sido estudiado por diversos autores y han surgido diferentes teorías al respecto, una de ellas es la teoría de roles que señala que existe una relación entre los dominios laborales y familiares y cuando entran en un conflicto de roles provoca una pérdida del balance de vida y trabajo (Khan, 1992). Otra teoría es la del Spillover en las que se describe como los sucesos positivos o negativos que suceden en una de las 
esferas de la vida como es el trabajo afectan a otras esferas vitales en este caso la familia (Piotrkowski, 1978).

Se debe agregar que Greenhaus y Allen (2011) manifiestan que el balance entre vida y trabajo, es la medida en que un individuo logra la eficacia y la satisfacción en el trabajo y en sus roles familiares Antes de la pandemia y del confinamiento las empresas ya trabajaban en políticas de balance entre vida y trabajo principalmente por las diferencias existentes al tener conviviendo en el trabajo tres generaciones con puntos de vista diferentes, un ejemplo de estas políticas es la de permitir un día al mes que alguien de la familia visite el trabajo o bien eventos fuera de la hora de trabajo para convivencia con la familia, por ejemplo: cenas, ferias, entre otras, las cuales eran muy eficaces sobre todo para los pertenecientes a la generación $\mathrm{Y}$ para quienes es muy importante encontrar el equilibrio entre su trabajo y su vida personal (Hernández y González, 2019)

\subsection{Retención de Personal}

La crisis provocada con el confinamiento y la pandemia ha dejado un panorama desconocido para las organizaciones, esto los ha orillado a redefinir sus estrategias y buscar reinventarse. Uno de los retos de la "nueva normalidad" para estas empresas es retener al personal no perderlo por falta de estrategias adecuadas en esta etapa en la que se encuentra el país donde el home office es una solución para salir adelante, aparecen las cargas excesivas de trabajo que están provocando serios problemas de salud a su personal. Una de las soluciones a esta problemática es reforzar el salario emocional que se le proporciona en este tiempo de coronavirus, el salario emocional son todas las alternativas que se ofrecen a los trabajadores para mantenerlos en el trabajo, como un horario flexible, mejor clima laboral y en estos tiempos prácticas de home office y balance entre vida y trabajo (Nieves, 2020).

Para Hernández y González (2019) la retención del talento son las actividades y prácticas que la administración realiza para mantener a las personas en su puesto de trabajo. Ante la situación actual retener al personal es un desafío para la empresa, la solución más rápida sería pagar más, sin embargo no todas las organizaciones pueden hacerlo, según Deloitte (2020) para los empleadores una solución es ofrecer a sus trabajadores la oportunidad de trabajar de forma remota, lo que se conoce como flexibilidad espacial de home office.

\subsection{Generación "Y"}

Una generación es el grupo de personas que nacieron en un mismo período de tiempo y viven experiencias similares y son testigos de los mismos hechos históricos (Kupperschmidt, 2000). Otra definición señala que es un grupo de edad que comparte a lo largo de la historia un conjunto de experiencias formativas que los distinguen de generaciones anteriores (Ogg y Bonvalet, 2006).

La razón para estudiar las generaciones se debe a que se desarrolla una personalidad generacional que determina valores, creencias, expectativas de trabajo, qué entorno laboral los motiva y retiene en la empresa y es por ese motivo que la generación $Y$ ha sido ampliamente estudiada, actualmente están en el rango de 24 a 30 años de edad, nacieron con la tecnología por lo que se les considera nativos digitales, por este motivo no es difícil para ellos el uso de las plataformas digitales para trabajar a distancia, es algo cómodo y natural a diferencia de las generaciones anteriores, son buenos con las estrategias digitales y por ello organizan mejor su tiempo, sin embargo para ellos el home office les impide tener la libertad para viajar, salir con amigos, disfrutar de su libertad (Golik, 2013, Silvestre y Cruz, 2016).

Los individuos pertenecientes a la generación Y esperan trabajos retadores, en donde se les valore y que tenga un significado, buscan un balance entre vida y trabajo y la oportunidad de realizar su trabajo fuera de las oficinas corporativas, que se valoren sus opiniones, y en la medida en que esto se cumpla permanecerán en su trabajo (Butts et al., 2013).

\subsubsection{Género}

El género según Butler (1990) es la resultante del proceso a través del cual cada persona recibe un significado cultural. Por su parte, Lamas (1996) 
manifiesta que es el conjunto de creencias y representaciones sociales que se presentan en una sociedad en función de la diferencia anatómica entre hombres y mujeres, que define su función en la sociedad, en el trabajo y otras áreas. En este aspecto hombres y mujeres se encuentran haciendo home office sin embargo la carga de trabajo de la mujer es mayor que la de los hombres debido a que además de su trabajo tienen la carga del hogar, el $40 \%$ de las mujeres deben cuidar a otras personas a diferencia de los hombres (21\%), sin embargo, a pesar de la situación, las mujeres que practican home office tienden a permanecer en sus puestos de trabajo por las ventajas que ofrece esta práctica (Heras, 2020).

Por otro lado, desde la perspectiva de hombres y mujeres, los roles que se desempeñan en el trabajo y en lo personal difiere por cuestiones de género, las prácticas de balance entre vida y trabajo se suponen son iguales para ambos, especialmente para estas últimas que a pesar de cumplir con los diferentes roles de trabajadora, madre, esposa, entre otros, provocan que se pierda la línea entre lo laboral y lo personal, es por ellos que las empresas debe cumplir con políticas que le permitan encontrar este balance (Tapia et al., 2016).

En un estudio realizado a 154 estudiantes universitarios en el Noreste de México sobre el trabajo a distancia y la retención de personal se encontró que $42 \%$ de los encuestados contestaron que era muy importante poder hacer home office para permanecer en su trabajo y el $86 \%$ que era muy importante tener políticas de balance entre vida y trabajo para quedarse en su empleo actual (Martínez, Luna y Patiño, 2020).

Adicionalmente, la empresa Deloitte (2019) aplicó una encuesta en 42 países a 13,416 jóvenes que nacieron entre 1983 y 1994 para conocer los factores que los motivan a permanecer en su trabajo, y el $84 \%$ afirmó que las políticas de balance entre vida y trabajo son importantes para seguir en su empresa actual. Otro estudio fue hecho por el Work Institute (2018), se realizó en 15 países a 10,455 jóvenes con edades entre 25 y 35 para determinar los factores de retención más importantes para ellos, y se encontró que un $49 \%$ permanecía en sus trabajos si su empresa les ofrecía la oportunidad de hacer home office.

\section{Método}

El enfoque utilizado en esta investigación es de corte cuantitativo con un diseño transversal y no experimental, de alcance descriptivo. La muestra es de tipo no probabilístico incidental. Se aplicó un cuestionario a 106 participantes de los cuales, como se muestra en la tabla 2, el $51 \%$ fueron mujeres y el $49 \%$ hombres cuyas edades fluctuaron entre 24 y 28 años egresados, con desempeño en los últimos 5 años en las áreas de: manufactura, servicios, comercial, educación y gubernamental del Noreste de Nuevo León, México. Con respecto al tamaño de la muestra, Cervantes (2005), sugiere la relación que debe haber entre el número de ítems de una encuesta tipo Likert y el tamaño de la muestra, se recomienda que para 20 ítems sea de entre 5 y 20 sujetos o encuestas por ítems, o bien entre $100 \mathrm{y}$ 400 mediciones, por lo que 106 mediciones se pueden usar para un estudio.

Para llevar a cabo esta investigación se diseñó un instrumento de 37 ítems de tipo Likert denominado factores de retención de personal compuesto con las variables de retención de personal, sentido de pertenencia, flexibilidad de horario, home office y balance entre vida y trabajo la cual está compuesta de ítems propios y adaptación de las encuestas de Zaid, et al., (2020); Sekyi et al., (2020); Park (2020); Madero (2019); Presbítero et al., (2016); Menezes y Kelliher (2016); Stravou y Ierodiakonou (2016) y Jurado (2014). En la cual no se excluyó ninguno de los ítems y se obtuvo un $\alpha=0.958$.

En esta investigación solo se estudiarán 3 variables, retención de personal como variable dependiente, home office y balance entre vida y trabajo como independientes respectivamente; del instrumento antes mencionado se tomaron 19 ítems de los cuales 7 son de la variable de retención de personal, 6 ítems para medir la variable home office y 6 ítems para balance entre vida y trabajo. La escala utilizada fue Likert en donde 1) es totalmente en desacuerdo; 2) en desacuerdo; 3) ni en desacuerdo, ni de acuerdo; 
4) de acuerdo y 5) totalmente de acuerdo. $\mathrm{El} \alpha=$ 0.917

El método de recolección de datos se realizó con la aplicación de la encuesta a

egresados de los últimos 5 años de los sectores de manufactura, servicios, comercial, educación y gubernamental, entre 24 y 28 años que la contestaron, el análisis de datos se realizó programa SPSS statistics v21 de donde se extrajeron los siguientes resultados para desahogar los objetivos planteados para esta investigación. Los datos presentados en la tabla 1 mencionan que el $51 \%$ de los egresados encuestados son mujeres y el $49 \%$ son hombres.

Tabla 1 Género de egresados encuestados

\begin{tabular}{clcccc}
\hline & Frecuencia & Porcentaje & Porcentaje válido & $\begin{array}{l}\text { Porcentaje } \\
\text { acumulado }\end{array}$ \\
\hline \multirow{3}{*}{ Válidos } & Mujer & 54 & 50.9 & 50.9 & 50.9 \\
& Hombre & 52 & 49.1 & 49.1 & 100.0 \\
& Total & 106 & 100.0 & 100.0 & \\
\hline
\end{tabular}

Fuente: Elaboración propia, SPSS v21.

En la tabla 3 se muestra que el $42 \%$ de los encuestados tienen 23 años, el $29 \%$ cuenta con 24 años, un $12 \%$ en el rango de los 25 años al igual que los de 26 años, un $6 \%$ con 27 años y un $5 \%$ con 28 años.

Tabla 2. Rango de edades de los encuestados

\begin{tabular}{lcccc}
\hline Edad & Frecuencia & Porcentaje & Porcentaje válido & Porcentaje acumulado \\
\hline 23 & 42 & 39.6 & 39.6 & 39.6 \\
24 & 29 & 27.4 & 27.4 & 67.0 \\
25 & 12 & 11.3 & 11.3 & 78.3 \\
26 & 12 & 11.3 & 11.3 & 89.6 \\
27 & 6 & 5.7 & 5.7 & 95.3 \\
28 & 5 & 4.7 & 4.7 & 100.0 \\
Total & 106 & 100.0 & 100.0 & \\
\hline
\end{tabular}

Fuente: elaboración propia, SPSS v21

\section{Resultados}

En el tiempo actual, debido a la pandemia por COVID-19 el home office se ha convertido en un elemento clave para que las empresas sigan operando, aunado a esto las nuevas reformas en la Ley Federal del Trabajo ofrecen a los empleados que trabajan en esta modalidad beneficios que les permiten un mayor balance entre vida y trabajo, con la contingencia sanitaria se demostró que el home office es una forma eficiente de trabajo y de retener al personal, sin embargo es de relevancia saber cómo impacta esta práctica a hombres y mujeres de la generación $\mathrm{Y}$ para mantenerse en su puesto, debido a que el estar trabajando en el entorno familiar hace que se pierda la línea que divide al lo laboral de lo personal (Roncal, 2021). Para desahogar las hipótesis de investigación planteadas se realizaron estadísticos descriptivos, pruebas " $t$ " student, se utilizó este estadístico ya que se compararon las medias de dos grupos, hombres y mujeres de la generación 
Y, para usar este estadístico se deben cumplir dos condiciones, primero hace una prueba de normalidad Kolmogorov-Sminrov para determinar si la distribución es normal, los resultados fueron los siguientes, para la retención de personal se obtuvo un p-valor= 0.60 , para el home office 0.482 y para balance entre vida y trabajo $\mathrm{p}$-valor $=0.61$. Como el valor de $p>0.05$ hay evidencias para determinar que la muestra sigue una distribución normal con un nivel de significación del $5 \%$.

La segunda condición es determinar la homogeneidad de la varianza, para esto se uso utilizó la prueba de Levene de la cual se muestran resultados en la tabla $3 y$ en la tabla 4. En la tabla 3 para la variable de home office se obtuvo una $\mathrm{F}=1.876$ con una $\mathrm{p}>0.05$ por lo que las varianzas se consideren iguales, en cuanto a la variable balance entre vida y trabajo la significancia es p>0.05 por lo que se asumen varianzas iguales.

Los resultados en la tabla 3 muestran que existe una media entre las mujeres con respecto a la retención de personal (3.85) y en hombres (4.05) y en Home Office se encontró una media en mujeres (3.25) y hombres (3.83). La prueba de Leven para home office es p-valor> $0.05(0.174)$ en la tabla del $t$ student se muestra la comprobación de la igualdad de la varianza, y un nivel de significancia de $\mathrm{p}>0.05$ (0.004) la cual es positiva con respecto a la escala de Likert utilizada.

Tabla 3. Estadísticos de grupos por género con respecto al home office

\begin{tabular}{lllccc}
\hline & Género & N & Media & $\begin{array}{c}\text { Desviación } \\
\text { típ. }\end{array}$ & $\begin{array}{c}\text { Error típ. de } \\
\text { la media }\end{array}$ \\
\hline \multirow{2}{*}{ Retención } & Mujer & 54 & 3.8545 & .76277 & .10380 \\
& Hombre & 52 & 4.0522 & .55870 & .07748 \\
\hline Home & Mujer & 54 & 3.2562 & 1.09157 & .14854 \\
Office & Hombre & 52 & 3.8365 & .91614 & .12705 \\
\hline
\end{tabular}

Prueba " $t$ " para variables independientes entre retención y home office

$\begin{array}{cc}\begin{array}{c}\text { Prueba de } \\ \text { Levene }\end{array} & \text { Prueba T para la igualdad de medias }\end{array}$

\begin{tabular}{|c|c|c|c|c|c|c|c|c|c|}
\hline \multirow[b]{3}{*}{ Retención } & \multirow[t]{2}{*}{$\mathrm{F}$} & \multirow[t]{2}{*}{ Sig. } & \multirow[t]{2}{*}{$\mathrm{t}$} & \multirow[t]{2}{*}{ gl } & \multirow[t]{2}{*}{$\begin{array}{c}\text { Sig. } \\
\text { (bilateral) }\end{array}$} & \multirow[t]{2}{*}{$\begin{array}{l}\text { Diferencia } \\
\text { de medias }\end{array}$} & \multirow[t]{2}{*}{$\begin{array}{l}\text { Error típ. } \\
\text { de la } \\
\text { diferencia }\end{array}$} & \multicolumn{2}{|c|}{$\begin{array}{c}95 \% \text { Intervalo de } \\
\text { confianza para la } \\
\text { diferencia }\end{array}$} \\
\hline & & & & & & & & Inferior & Superior \\
\hline & 3.216 & .076 & -1.518 & 104 & .132 & -.19770 & .13027 & -.45604 & .06064 \\
\hline Home Office & 1.876 & .174 & -2.959 & 104 & .004 & -.58037 & .19611 & -.96926 & -.19147 \\
\hline
\end{tabular}

Para desahogar las hipótesis y determinar el balance entre vida y trabajo en la retención de personal de hombres y mujeres de la Generación Y, se utilizó una prueba " $t$ " student para muestras independientes, en la tabla 4 se muestra que para la variable de retención en el caso de las mujeres el resultado fue una media de 3.85 contra el de los hombres; 4.05, en de balance entre vida y trabajo en el caso de la mujeres se obtuvo una media de 3.79 y en el de los hombres 4.05 , se obtuvo una $\mathrm{p}<0.05(0.156)$ la cual no fue significativa para esta variable.

Fuente: Elaboración propia, SPSS v21 
Tabla 4. Estadísticos de grupo por género para balance entre vida y trabajo

\begin{tabular}{lccccc}
\hline & Género & $\mathrm{N}$ & Media & Desviación típ. & $\begin{array}{c}\text { Error típ. de la } \\
\text { media }\end{array}$ \\
\hline Retención & Mujer & 54 & 3.8545 & .76277 & .10380 \\
Balance entre & Hombre & 52 & 4.0522 & .55870 & .07748 \\
vida y trabajo & Hombre & 54 & 3.7963 & .90460 & .12310 \\
\hline
\end{tabular}

Prueba " $t$ " para variables independientes entre retención y balance entre vida y trabajo

\begin{tabular}{|c|c|c|c|c|c|c|c|c|c|}
\hline & \multicolumn{2}{|c|}{$\begin{array}{l}\text { Prueba de } \\
\text { Levene }\end{array}$} & \multicolumn{7}{|c|}{ Prueba T para la igualdad de medias } \\
\hline & \multirow[t]{2}{*}{$F$} & \multirow{2}{*}{ Sig. } & \multirow{2}{*}{$\mathrm{t}$} & \multirow{2}{*}{ gl } & \multirow{2}{*}{$\begin{array}{c}\text { Sig. } \\
\text { (bilateral) }\end{array}$} & \multirow{2}{*}{$\begin{array}{l}\text { Diferencia } \\
\text { de medias }\end{array}$} & \multirow{2}{*}{$\begin{array}{l}\text { Error típ. } \\
\text { de la } \\
\text { diferencia }\end{array}$} & \multicolumn{2}{|c|}{$\begin{array}{c}\text { 95\% Intervalo de } \\
\text { confianza para la } \\
\text { diferencia }\end{array}$} \\
\hline & & & & & & & & $\begin{array}{l}\text { Inferior } \\
\text { Inferior }\end{array}$ & $\begin{array}{l}\text { Superior } \\
\text { Superior }\end{array}$ \\
\hline Retención & 3.216 & .076 & -1.518 & 104 & .132 & -.19770 & .13027 & -.45604 & .06064 \\
\hline $\begin{array}{l}\text { Balance entre vida y } \\
\text { trabajo }\end{array}$ & .064 & .801 & -1.430 & 104 & .156 & -.25499 & .17834 & -.60864 & .09867 \\
\hline
\end{tabular}

Fuente: elaboración propia, SPSS v21

\section{Conclusiones}

Uno de los principales retos de las organizaciones es retener al personal en estos tiempos donde con la evolución de la tecnología se han implementado prácticas de home office que permiten que hombres y mujeres trabajen a distancia, sin embargo esto ha provocado que se pierda el balance entre vida y trabajo puesto que no hay una división entre las actividades laborales y las personales, esto impacta de forma diferente a ambos géneros por la naturaleza de las obligaciones que cada uno de ellos tiene, pues históricamente las mujeres tienen además de su trabaja, la carga de las labores del hogar y el rol de mamá, lo que impacta la retención de personal entre hombres y mujeres, si la empresa no implementa políticas adecuadas para prevenirlo (Rodríguez et al., 2018).

Con base en la información encontrada se observa que la $\mathrm{H} 1$ se cumple puesto que para a los jóvenes de la generación $\mathrm{Y}$ es significativo poder trabajar a distancia para permanecer en la empresa . En diversos estudios de índole nacional e internacional se mostró que para la generación Y, uno de los factores que más los retiene en su trabajo, es poder practicar home office puesto que les permite manejar su tiempo eficientemente con respecto a los roles que tienen que realizar en su día a día (Martínez et al.,2016; Work Institute, 2018; Deloitte, 2019).

En relación con el segundo objetivo de investigación no se encontró una diferencia significativa entre la retención y el balance entre vida y trabajo, en las comparación de medias esta fue positiva lo que concuerda con lo encontrado en los estudios encontrados en la literatura en la cual en estudios realizados en diversos países y en México los jóvenes entre 23 y 29 años permanecen en las empresas que tienen políticas entre balance entre vida y trabajo.

Con respecto a lo anterior, Silvestre y Cruz (2016) señalan que la generación $\mathrm{Y}$ son los nacidos entre 1983 y 1999. Existen diferencias entre los pertenecientes de la generación Y, los mayores rondan los 38 años y los más jóvenes 22 años, estos últimos están en un período de transición generacional con los llamados centennials, los que se encuentran entre 22 y 24 años tienen más similitudes con los centennials 
que con sus cohortes, algo en que coinciden estas dos generaciones es que son role-hopping es decir que buscan explorar otras opciones dentro de la misma empresa, es decir cambiar de puestos y crecer aunque tengan que invertir parte de su tiempo personal para lograrlo (Solís et al., 2020).

Lo anterior será de utilidad para que las organizaciones implementen políticas que les permitan a los sus empleados mantener un balance entre su vida laboral y personal y más en estos tiempos de pandemia por COVID-19 en donde las empresas se vieron obligadas de pasar del trabajo en sus instalaciones a un trabajo a distancia en donde los roles como trabajador y personal se mezclaron en un mismo entorno, y como se encontró en la literatura en donde se encontró que se trabajan más horas desde la pandemia.

La teoría de empresas flexibles de Atkinson (1987) está asociada al home, señala que las empresas buscan crear puestos a distancia aprovechando las habilidades de sus empleados en la tecnología, en la actualidad esta teoría está muy actual ante la pandemia que ha provocado que las empresas sean flexibles y busquen las condiciones para adaptar sus puestos al home office.
Con respecto a los anterior, el conocer estos datos permitirá a prepararse para cumplir con la reforma en el artículo 311 de la Ley Federal del Trabajo publicada el 11 de enero de 2021 en donde queda definido el teletrabajo y las condiciones en las que se debe llevar a cabo.

Cabe mencionar que los resultados de este trabajo de investigación no se pueden generalizar, debido que hay una limitación importante en la muestra utilizada puesto que para el análisis se tomó específicamente de personas de la generación Y. Para poder generalizar los resultados de este trabajo se deberá tomar en cuenta las limitaciones en futuros estudios. Este estudió puede aplicarse a otras líneas de investigación como estudios de otras generaciones, también aplicarse a puestos gerenciales y de puestos de operarios para contrastar resultados. 


\section{REFERENCIAS}

Álvarez, H. (2020). Del recurso al teletrabajo como medida de emergencia al futuro del trabajo a distancia. Revista de Relaciones Laborales, 43(1), 175-201. Obtenido de www.dialnet.uniroja.es

Atkinson, J. (1985). Flexiblity, uncertainty and manpower management. UK: University of Sussex.

Butler, J. (1990). Gender Trouble: feminism and the subversion of identity. United States: Routledge.

Butts, M., Casper, W., \& Yang, T. (2013). How important are work-family support policies? A metaanalytic investigation of their effects on employee outcomes. Journal of Applied Psychology, 98(1), 1-25. Obtenido de https://psycnet.apa.org/record/2012-28744-001

Candela, R., \& Dabos, G. (2017). Gestión individual del equilibrio entre el trabajo y la vida personal: revisión e integración de la literaura. Revista Facultad de Ciencias Económicas: investigación y reflexión, XXV(1), 219-242. Obtenido de https://www.redalyc.org/pdf/909/90949035015.pdf

Cervantes, V. H. (2005). Interpretaciones del coeficiente alpha de Crobanch. avances en medición. $\begin{array}{lllll}\text { Research } & \text { Gate, } & 3(1), & 9-28 . & \text { Obtenido de }\end{array}$ http://www.humanas.unal.edu.co/psicometria/files/1113/8574/8604/Articulo_1_Alfa_de_Cr onbach_9-28_2.pdf

Deloitte (2019). Obtenido de https://www2.deloitte.com/es/es/pages/humancapital/articles/guiaatraer-fidelizar-talento-millennial.html

Fócil, M. (2020). Teletrabajo en el sector público en México: ¿Llegó pra quedarse? Revista Buen Gobierno, 1(1), 1-27. doi:http://dx.doi.org/1035247/buengon_29_04

Golik, M. (2013). Las expectativas de equilibrio entre vida laboral y vida privada y las elecciones laborales de la nueva generación. Cuadernos de Administración, 26(46), 107-133. Obtenido de https://www.redalyc.org/pdf/205/20527100005.pdf

Gómez, G. (2020). Consecuencias de las propuestas educativas en la salud socioemocional de diversos actores educativos. Revista Latinoamericana de Estudios Educativos, L(I), 241-248. Obtenido de https://www.redalyc.org/jatsRepo/270/27063237005/html/index.html

Greenhaus, J., \& Allen, T. (2011). Work-family balance: a review and extensión of the literature. Washington, DC: American Psychological Associations.

Hernández, A., \& González, J. (2019). Estructura interna de la escala de conciliación vida-trabajo para mujeres asalariadas del noreste de México. Psicogente, 22(42), 1-18. Obtenido de www.scielo.org.co

Jurado, A. (2014). Los niveles de pertenencia en un grupo de profesores bajo contratación laboral simulada en distintas organizaciones Guatemaltecas. (Tesis de Maestría). Universidad Rafael landívar, Guatemala de Asunción.

Khan, W. A. (1992321-349). To be fully there: psychological presence at work. Human Relations, 45(4). Obtenido de https://journals.sagepub.com/doi/10.1177/001872679204500402

Kupperschmidt, B. (2000). Multigeneration employees: strategies for effective management. The Healt Care Manager, 19(1), 65-76. Obtenido de www.researchgate.net 
Lamas, M. (1996). El género: La construcción cultural de la diferencia sexual. Ciudad México: Editorial Porrúa.

LFT. (2019). Ley Federal del Trabajo. Art. 311. México:Porrúa

Martin, V., \& Reyes, G. (2020). Desafíos y nuevos escenarios gerenciales como parte de la herencia del Covid-19. Revista Venezolana de Gerencia, 25(90), 710-719. Obtenido de https://www.redalyc.org/jatsRepo/290/29063559019/29063559019.pdf

Martínez, R., Luna, S., \& Patiño, I. (2020). Generación Y de la UANL: El grado de importancia de los factores personales y flexibilidad laboral para permanecer en el trabajo. VinculaTégica, 6(1), 319-329. Obtenido de www.web.facpya.uanl.mx

Menezes, L., \& Kelliher, C. (2016). Flexible working individual perfomance and employee attitudes: comparing formal and infrmal arrangements. Human Resource Management, 56(6), 10511070.

Menezes, L., \& Kelliher, C. (2016). Flexible working individual perfomance, and employee attitudes: comparing formal and informal arrangements. Human Resources Management, 56(6), 10511070. Obtenido de https://www.wiley.com

Heras, M. (2021). Teletrabajo en tiempos de COVID-19. Recuperado de https://mireialasheras.com/research-reports/teletrabajo-contenido-en-espanol-form/

Nieves, L. (2020). Diseño de la herramienta salario emocional para el mejoramiento de la calidad de vida de los empleados del colegio el liceo paraíso de los niños en la ciudad de Barrancabermeja. (Tesis de Maestría). Universidad de Cooperativa de Colombia, Barrancabermeja.

OECD. (2020). Medidas políticas clave de OCDE ante el coronavirus (COVID-19). Obtenido de www.oecd.org

Ogg, J., \& Bonvalet, C. (2006). The baby bommer generation and the birth cohort Of 1945-1954: s european perspective. Paper presented at the ESGR-AHRC seminar, 1-18. Science weeks in March, 2006 on the Europan

Piotrkowski, C. (1978). Work and family system: a naturalistic study of workingclass families. New York: Free Press.

Rodríguez, M., Dabos, G., \& Rivero, A. (2018). Implementación de políticas de conciliación trabajovida en pequeñas y medianas empresas: un estudio de casos múltiples en la industria del software. Estudios Gerenciales, 34(147), 172-189. Obtenido de http://www.scielo.org.co/scielo.php?script=sci_arttext\&pid=S0123-59232018000200172

Roncal, X. (2021). Teletrabajo y capitalismo de vigilancia. Telos, 23(1), 177-192. Obtenido de https://www.redalyc.org/jatsRepo/993/99365404014/99365404014.pdf

Silvestre, E., \& Cruz , M. (2016). Conociendo la próxima generación de estudiantes universitarios dominicanos a través de redes. Ciencia y Sociedad, 41(3), 475-503. Obtenido de: https://revistas.intec.edu.do/index.php/ciso/citationstylelanguage/get/acs-n

Solis, F., Cisneros, G.,Guaderrama, K. \& Portillo, M. (2020). Motivaciones de las generaciones millennial y centennial para la creación de nuevas empresas. Revista y Administración y 
Organizaciones, 23(45), 84-99. Obtenido de: https://rayo.xoc.uam.mx/index.php/Rayo/article/view/440

Statista. (2021). Obtenido de https://es.statista.com/estadisticas/1147384/porcentaje-empleadoshome-office-mexico/

Tapia, P., Saracostti, M., \& Castillo, L. (2016). Balance entre familia y trabajo: un análisis comparativo de Chile y Francia desde una normativa internacional hacia regulaciones laborales. Revista Ius et Praxis, 22(1), 493-524. Obtenido de https://scielo.conicyt.cl/pdf/iusetp/v22n1/art14.pdf

Tello, C. (2020). Género y trabajo en tiempos del COVID-19: una mirada desde la interseccionalidad. Revista Venezolana de Gerencia, 25(90), 389-393. Obtenido de

https://www.redalyc.org/jatsRepo/290/29063559020/html/index.html

Work Institute. (s.f.). Obtenido de

https://cdn2.hubspot.net/hubfs/478187/2018\%20Retention\%20Report/Work\%20Institute\% 202018\%20Retention\%20Report\%20043018\%20-

\%20Final.pdf?_hssc $=163589856.1 .1586337893925 \&$ _hstc $=163589856 . c 45 \mathrm{~d} 2 \mathrm{ea} 23 \mathrm{e} 5816$ 1ca2d44e51e0ea72a6.1586337893924.1586337893924.1586337 